\title{
NOTE ON THE
}

\section{REAL INFLEXIONS OF PLANE CURVES*}

\section{CHARLOTTE ANGAS SCOTT}

The determination of the possible situation of the real inflexions of a curve $u=0$ is often practically very difficult. The work can sometimes be simplified by means of the fact that real inflexions lie only in the region for which $u_{11} u_{22}-u_{12}^{2}$ is zero or negative, where $u_{11}, u_{22}, u_{12}$ are written for $\partial^{2} u / \partial x^{2}, \partial^{2} u / \partial y^{2}, \partial^{2} u / \partial x \partial y$. This expression, $G$, depends on the relation of the curve $u=0$ to the line $z=0$; a corresponding expression can of course be obtained with reference to any line, and for a real inflexion every such expression must either be zero or have a negative value.

The proof is extremely simple. The polar conic of any point is

$$
x^{2} u_{11}+y^{2} u_{22}+z^{2} u_{33}+2 y z u_{23}+2 z x u_{31}+2 x y u_{12}=0 ;
$$

for a double point or a point of inflexion this represents a pair of straight lines, namely, the tangents at the double point, or the inflexional tangent together with another line which does not pass through the point of inflexion. This line-pair will be imaginary if any one such function as $u_{11} u_{22}-u_{12}^{2}$ is positive; it will be real if no one of these functions is positive, that is, if every one is either zero or negative. This is ensured if any one such function is negative, or if two are zero.

The expression $G$ is regularly used in discriminating between real and imaginary tangents at a node, but it does not appear to have been used in considering the possibility of real inflexions. If we equate it to zero, we obtain a curve $G=0$, derived from $u$ and the line $z$, the locus of points whose polar conics meet $z$ in coincident points. If $z$ is the line at infinity, $G$ is the locus of points whose polar conics are parabolas, including parallel straight lines and coincident straight lines, in which last case the points are cusps or higher singularities. $\dagger$ The curve $G$ divides the plane into regions; the polar conics of points in the

* Presented to the Society December 28, 1901. Received for publication August 16, 1902.

† The higher singularities here referred to are double points with coincident tangents ; multiple points of order higher than 2 lie on $G=0$ for a different reason, namely, on account of the vanishing of $u_{11}, u_{22}$, etc. 
region in which the expression $G$ is positive meet the line $z$ in imaginary points (ellipses and imaginary straight lines) and the polar conics of points in the region defined by $G$ negative meet $z$ in real points (hyperbolas and real straight lines).

It would seem that the curve thus derived from $u$ and a line, as the locus of points whose polar conics touch the line, or as the envelope of the so-called second polars of points on the line, merits consideration. The name "diacritic" suggests itself; $G$ is the diacritic (or diacritic curve) of $z$ with respect to $u$. If $u$ be a cubic, $G$ is simply the "Poloconik" of the line.

The diacritic of $z$ is the same with respect to all curves of the family $u=k$ ( $u=k z^{n}$ in homogeneous coördinates) ; it separates the region in which isolated points can lie from the region in which the other nodes (and all real inflexions) are to be found, and thus it must plainly be of importance in the consideration of the critic centres of this pencil. These critic centres are the points common to the first polars of points at infinity, $u_{1}+\lambda u_{2}=0$; for this linear system they take the place of an envelope for a more general system, while the diacritic is the envelope of the second polars of points at infinity, $u_{11}+2 \lambda u_{12}+\lambda^{2} u_{22}=0$.

The diacritic is the locus of points of contact of curves $u_{1}=$ constant with curves $u_{2}=$ constant. This fact is also expressed in the more general statement that the increment of $u_{1}$ along the curve $u_{2}=0$ depends at any point on the sign of $G$ (since $\delta u_{1}=u_{11} \delta x+u_{12} \delta y$, where $u_{12} \delta x+u_{22} \delta y=\delta u_{2}=0$ ), and consequently changes sign only when $G=0$. This shows that in passing along $u_{2}=0$ from one critic centre to the next we pass over $G$; in general the critic centres are alternately isolated points and nodes with reai tangents.

Bryn Mawr College, Pennsyluania. 